\title{
Patient after extensive crushing injury of the lower limb with subacute stent thrombosis
}

\author{
Corresponding author: \\ Michał Kasprzak, Department \\ of Cardiology and Internal Medicine, \\ Collegium Medicum in Bydgoszcz, \\ Nicolaus Copernicus University \\ Poland,medkas@o2.pl \\ Medical Research Journal 2021; \\ Volume 6, Number 3, 270-273 \\ 10.5603/MRJ.a2021.0031 \\ Copyright (C) 2021 Via Medica \\ ISSN 2451-2591 \\ e-ISSN 2451-4101
}

\begin{abstract}
Percutaneous transluminal coronary angioplasty with stent implantation is a basic life-saving treatment of stenotic lesions causing acute coronary syndromes. Stent thrombosis is one of the most serious complications of coronary angioplasty, strongly associated with recurrent myocardial infarction and high mortality. Many factors were identified as increasing the incidence of stent thrombosis including bleeding and inflammation. I am presenting a case of a 54-year-old man after extensive crushing injury of the lower limb with simultaneous stent thrombosis in two recently implanted stents. As a preventive measure for stent thrombosis novel potent antiplatelet agents may be a reasonable choice even for patients with high bleeding risk

Key words: stent thrombosis, crushing injury, inflammation, bleeding
\end{abstract}

Med Res J 2021; 6 (3): 270-273

\section{Introduction}

Percutaneous transluminal coronary angioplasty (PTCA) with new-generation drug-eluting stent (DES) implantation has become the most frequently performed therapeutic procedure in medicine. It is a basic treatment of flow-limiting coronary stenosis both in acute and chronic coronary syndromes. One of the most serious complications of coronary stent implantation is stent thrombosis (ST). ST is usually causing ST-elevation myocardial infarction (MI) associated with a high mortality rate. This detrimental condition may occur any time from immediately after the procedure to several years thereafter. ST categorized as subacute (2-30 days after PTCA) carries the highest mortality risk [1].

\section{Case report}

A 54-year-old patient was admitted to the hospital emergency department due to an extensive crushing injury of the right leg as a result of a farm accident. The patient was qualified for immediate surgery. Debridement of the left lower leg wound, tibial stabilization with an external stabilizer, intraoperative application of gentamicin and thrombectomy of the right posterior tibial artery were performed. On admission laboratory tests showed the following deviations: white blood cell count (WBC) - $19.5 \times 10^{9} / \mathrm{L}$, C-reactive protein (CRP) $-8 \mathrm{mg} / \mathrm{L}$. Postoperatively anaemia with haemoglobin (Hb) concentration $10.5 \mathrm{~g} / \mathrm{dL}$ and significantly elevated troponin I levels determined by the high sensitive method (hsTPI) - $1853 \mathrm{ng} / \mathrm{L}$ were observed. In the next days of hospitalization, elevated WBC $\left(11.7 \times 10^{9} / \mathrm{L}\right)$, increase in CRP (340 mg/L), further anaemia $(\mathrm{Hb} 8.9 \mathrm{~g} / \mathrm{dL})$ and an increase in hsTPI (4640 ng/L) were observed.

On the second and third day of hospitalization, the patient periodically reported mild resting pressure-type chest pain. Detailed medical history revealed nicotinism, hypertension and exertional chest pain for several months. ECG showed: intermediate axis, sinus rhythm 96 bpm, Q waves in II, III, aVF, ST-segment depression and negative T waves in V4-V6 (Fig. 1). Echocardiography revealed left ventricular hypertrophy, moderate mitral regurgitation, moderate left ventricular systolic dysfunction with regional contractility disorders, and left ventricular ejection fraction of $38 \%$. A diagnosis of non-ST elevation MI was made, and the patient was qualified for coronarography. It revealed advanced three-vessel disease with $100 \%$ stenosis of the right coronary artery, $90 \%$ stenosis of proximal and $80 \%$ stenosis middle segment of left anterior descending artery 


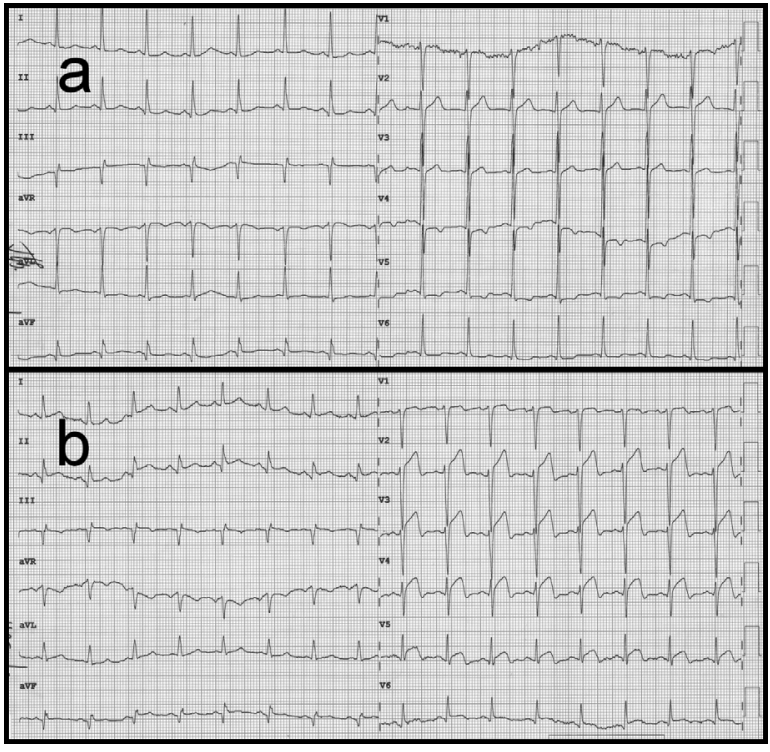

Figure 1. ECG: $a-4^{\text {th }}$ hospitalization day, $b-14^{\text {th }}$ hospitalization day; speed $25 \mathrm{~mm} / \mathrm{s}$

(LAD), $50 \%$ stenosis of the second diagonal branch, $70 \%$ ostial stenosis of the first septal branch and $90 \%$ stenosis of the second obtuse marginal branch (OM2) (Fig. 2). An immediate cardiac surgery consultation was performed to qualify for coronary artery bypass grafting. After a detailed explanation of treatment options, risks, anticipated benefits and limitations of heart surgery and PTCA the patient refused surgery and chose percutaneous treatment. Accordingly, PTCA OM2 was performed ad hoc by implanting DES Firehawk $2.5 \times 29 \mathrm{~mm}$ at pressure $14 \mathrm{~atm}$ with a good direct result (Fig. 2). Before angioplasty, loading doses of acetylsalicylic acid and clopidogrel were given. Ticagrelor was not used due to recent extensive surgical trauma and a significantly increased risk of bleeding. Two days later, the second stage of PTCA was performed with two DES implantation (Promus Premier $2.5 \times 32 \mathrm{~mm}$ at 12 atm in middle LAD and Synergy $3.0 \times 28 \mathrm{~mm}$ at $12 \mathrm{~atm}$ in proximal $\mathrm{LAD}$ ) and post-dilatation with non-compliant (NC) balloons Quantum Apex $3.5 \times 15 \mathrm{~mm}$ and $4.0 \times 12 \mathrm{~mm}$ up to $18 \mathrm{~atm}$ (Fig. 2). The course of hospitalization at the Cardiology Clinic was without complications. After obtaining microbiological results initial empirical antibiotic therapy was modified. Decreasing hsTPI (893 ng/L) and CRP (193 mg/L), low procalcitonin (PCT) concentration $(0.43 \mathrm{ng} / \mathrm{mL})$, stable $\mathrm{Hb}(9.2 \mathrm{~d} / \mathrm{dL})$ and WBC $\left(11.7 \times 10^{9} / \mathrm{L}\right)$ were observed. After an orthopaedic consultation, the patient was transferred to the Orthopaedics Clinic where surgical drainage of left lower leg wounds was performed. Due to anaemia $(7.2 \mathrm{~d} / \mathrm{dL})$, four units of packed red blood cells (PRBC) were transfused. Laboratory tests showed stable $\mathrm{Hb}(10.2 \mathrm{~g} / \mathrm{dL})$,
WBC $\left(10.6 \times 10^{9} / \mathrm{L}\right)$, increasing platelet count (PLT) $\left(466 \rightarrow 787 \rightarrow 1044 \times 10^{9} / L\right)$, low PCT, decrease in CRP (109mg/dL) and hsTPI (165 ng/L).

On the seventh day after being transferred from the Cardiology Clinic, the patient reported severe tearing pain in the chest radiating to the neck, lower jaw and left upper limb. ECG revealed ST-segment elevation in V2-V5 up to $3 \mathrm{~mm}$, with hyperacute $\mathrm{T}$ waves (Fig. 1). LAD stent thrombosis was suspected. The patient was transported to the cath lab immediately. Coronary angiography revealed stent thrombosis in both $L A D$ and OM2 (Fig. 2). A loading dose of ticagrelor and a bolus followed by an infusion of abciximab were given. Aggressive dilatations within LAD and OM2 stents with $\mathrm{NC}$ balloons Apollo $2.5 \times 12 \mathrm{~mm}$ and $3.0 \times 12 \mathrm{~mm}$ at pressures up to $30 \mathrm{~atm}$ were carried out. Restoration of flow, good angiographic effect and chest pain resolution were achieved.

In the following days, the local condition of the limb was rapidly deteriorating. A gradual worsening of the general condition was also observed. A decision was made to amputate the limb. Due to the recent acute coronary syndrome (ACS) and ST, the procedure was performed without interrupting antiplatelet therapy. Four PRBC units were transfused during the perioperative period and a transient increase in hsTPI up to $6294 \mathrm{ng} / \mathrm{L}$ without new ECG or echocardiography findings was observed (with a perioperative decrease in $\mathrm{Hb}$ to $7.4 \mathrm{~g} / \mathrm{dL}$ ).

After amputation further lowering of inflammation parameters and gradual improvement in the patient's condition was observed. Due to negative control cultures, antibiotic therapy was terminated. The patient was discharged home in good general condition 33 days after injury.

\section{Discussion}

In recent years, the development of new generation DES, improvement of implantation techniques and introduction in clinical practice novel potent antiplatelet drugs resulted in a significant reduction in the incidence of ST. Recent large-scale registries reported that with contemporary treatment ST occurs in less than $2 \%$ of patients [2, 3].

Many clinical, angiographic and procedural factors were proven to be related to an increased risk of ST [4]. Clinical factors with higher ST risk include premature antiplatelet treatment discontinuation, diabetes, renal failure, anaemia, impaired LVEF, malignancy, smoking, advanced age, antiplatelet drugs resistance. Angiographic and device-related factors predisposing to ST include ACS, complex lesion morphology, multiple lesions, stent under expansion, residual stenosis, vessel dissection, stenting in small vessels, suboptimal 


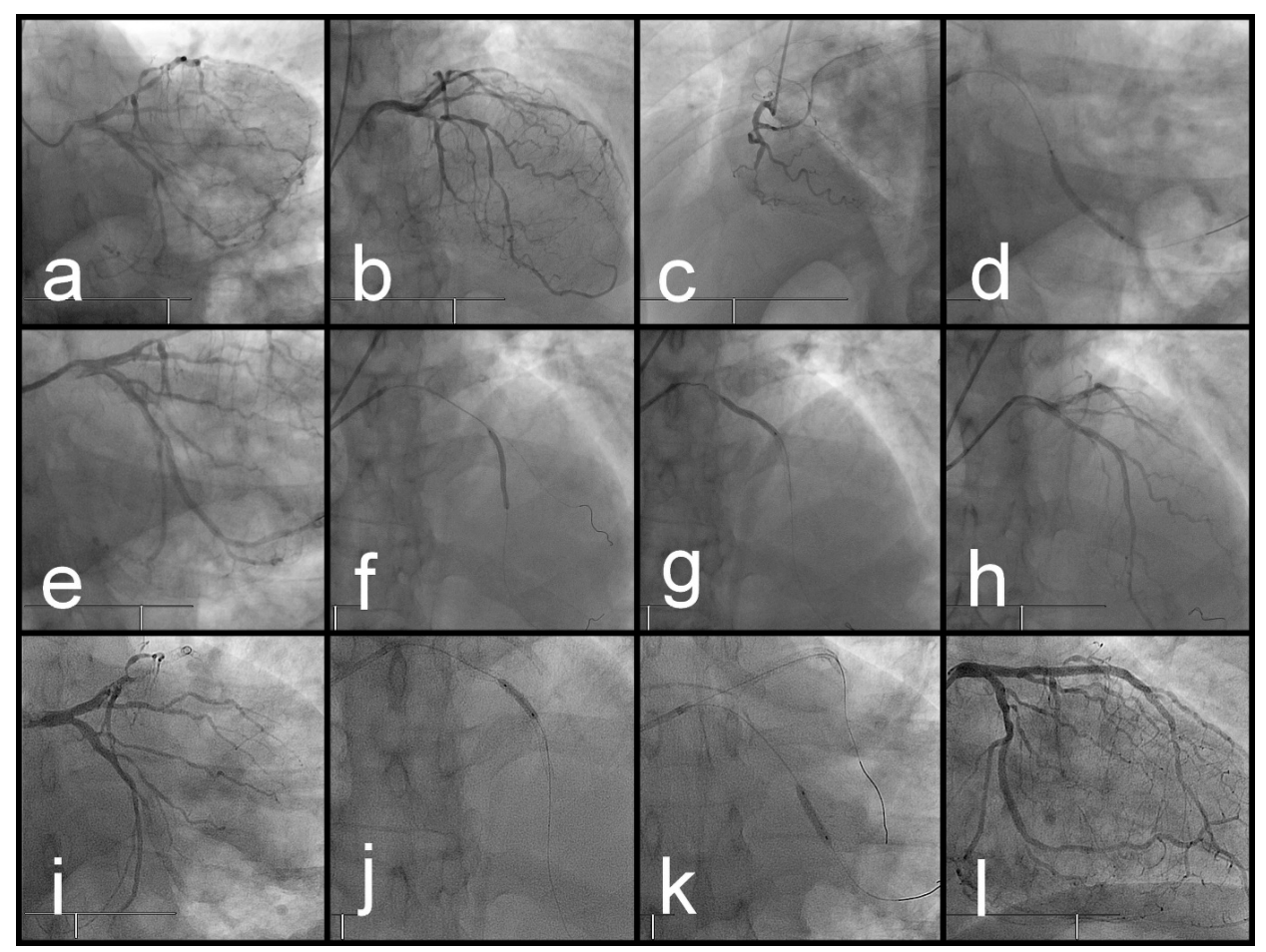

Figure 2. Coronary angiograms: a - pre-PTCA AP caudal view, $b-$ pre-PTCA AP cranial view, $c$ - pre-PTCA left anterior oblique view - RCA, d - OM2 stent implantation, e — post-PTCA OM2 AP caudal view, $f$ - middle LAD stent implantation, $g$ - proximal LAD stent implantation, $h$ - post-PTCA LAD AP cranial view, $\mathrm{i}$ - LAD and OM2 ST AP caudal view, j - LAD ST baloon angioplasty AP cranial view, $k$ - OM2 ST baloon angioplasty AP caudal view, I - final result after ST angioplasty right anterior oblique caudal view; AP — anterio-posterior, LAD — left anterior descending artery, OM2 - second obtuse marginal branch, PTCA — percutaneous transluminal coronary angioplasty, RCA — right coronary artery, ST — stent thrombosis

stenting result with the impaired coronary flow. The risk of ST depends also on the type of implanted stent. New-generation DES with enhanced biocompatibility exclusively sirolimus-analogue active drugs and thin struts have a significantly lower risk of stent thrombosis in comparison to both early-generation DES and BMS [5]. Accordingly, new-generation DES should be the default stent type for PTCA regardless of clinical presentation, lesion subtype, concomitant therapies, or comorbidities [6].

Several studies have reported an association between inflammatory cytokines concentrations and stent thrombogenicity [7-9]. Park et al reported that pre-procedural CRP was significantly associated with increased risks of ST, death, and MI [7]. Hwang et al. [8] reported a positive association between elevated levels of interleukin 6 and DES thrombosis. Katayama et al. have shown that in patients with MI who are treated with primary coronary stenting, inflammation indicators such as CRP and serum amyloid-A protein may be closely related to ST [9]. Some previous reports have shown that major bleeding may also be correlated with the development of ST [1]. This case report presented the patient with both high-grade inflammation and recurrent anemization.

In general, as a preventive measure against ST novel more potent P2Y12 inhibitors, namely ticagrelor and prasugrel, may be used. These drugs achieve a faster, greater and more consistent degree of P2Y12 inhibition as compared to clopidogrel. They are more effective in preventing early and late ST in patients with ACS [10, 11]. However, this comes at the cost of higher bleeding liability and previous intracranial haemorrhage or ongoing bleeds are contraindications for ticagrelor and prasugrel $[10,11]$. Due to recent trauma with peripheral vessel injury, the presented patient was initially assessed as ineligible for ticagrelor or prasugrel treatment.

Observational studies have shown a high risk of ST recurrence after the first episode [12,13]. Armstrong et al reported the cumulative hazard of angiographic definite recurrent ST $11 \%$ at 1 year and $20 \%$ at 5 years. The cumulative hazard of definite or probable recurrent ST was $16 \%$ at 1 year and $24 \%$ at 5 years. According to this registry, the risk of recurrence is highest in the first few months after the first event [12]. Both prasugrel and ticagrelor are associated with a significant reduction 
of first and recurrent ST as compared to clopidogrel $[10,13]$. Based on this data after ST occurrence patients previously on clopidogrel should be switched to ticagrelor or prasugrel if not contraindicated [6]. Bolus and infusion of glycoprotein Ilb/Illa receptor antagonist (abciximab, tirofiban or eptifibatide), potent antiplatelet drug and aggressive high-pressure balloon dilations are standard ST treatments [6]. In most cases, satisfactory results are obtained with balloon dilation and repeated stenting may be avoided. However, a new stent may be required to overcome edge-related dissections and adjacent lesions, or to optimize final results [6, 14]. Despite the high bleeding risk, in the face of ST in stents implanted both in LAD and OM2, the presented patient was given abciximab and was switched to ticagrelor. Subsequent leg amputation was carried out successfully on full dual antiplatelet therapy with reasonable blood loss.

In conclusion, ST is an important serious complication following angioplasty which prevention and treatment often require difficult clinical decisions with risk-benefit assessment. Novel potent antiplatelet agents may be a reasonable choice even for patients with high bleeding risk.

\section{Conclusions}

ST is an important serious complication following angioplasty which prevention and treatment often require difficult clinical decisions with risk-benefit assessment. As a preventive measure novel potent antiplatelet agents may be a reasonable choice even for patients with high bleeding risk.

Conflict of interest: None.

\section{References}

1. Heestermans AA, van Werkum JW, Zwart B, et al. Acute and subacute stent thrombosis after primary percutaneous coronary intervention for ST-segment elevation myocardial infarction: incidence, predictors and clinical outcome. J Thromb Haemost. 2010; 8(11): 2385-2393, doi: 10.1111/j.1538-7836.2010.04046.x, indexed in Pubmed: 20831622.
2. Byrne RA, Joner M, Kastrati A. Stent thrombosis and restenosis: what have we learned and where are we going? The Andreas Grüntzig Lecture ESC 2014. Eur Heart J. 2015; 36(47): 3320-3331, doi 10.1093/eurheartj/ehv511, indexed in Pubmed: 26417060.

3. Tada T, Byrne RA, Simunovic I, et al. Risk of stent thrombosis among bare-metal stents, first-generation drug-eluting stents, and second-generation drug-eluting stents: results from a registry of $18,334 \mathrm{pa}-$ tients. JACC Cardiovasc Interv. 2013; 6(12): 1267-1274, doi: 10.1016/j. jcin.2013.06.015, indexed in Pubmed: 24355117

4. Räber L, Magro M, Stefanini GG, et al. Very late coronary stent thrombosis of a newer-generation everolimus-eluting stent compared with early-generation drug-eluting stents: a prospective cohort study. Circulation. 2012; 125(9): 1110-1121, doi: 10.1161/CIRCULATIONAHA.111.058560, indexed in Pubmed: 22302840.

5. Palmerini T, Benedetto $U$, Biondi-Zoccai $G$, et al. Long-term safety of drug-eluting and bare-metal stents: evidence from a comprehensive network meta-analysis. J Am Coll Cardiol. 2015; 65(23): 2496-2507, doi: 10.1016/j.jacc.2015.04.017, indexed in Pubmed: 26065988.

6. Neumann FJ, Sousa-Uva M, Ahlsson A, et al. ESC Scientific Document Group, ESC Scientific Document Group. Considerations for the choice between coronary artery bypass grafting and percutaneous coronary intervention as revascularization strategies in major categories of patients with stable multivessel coronary artery disease: an accompanying article of the task force of the 2018 ESC/EACTS guidelines on myocardial revascularization. Eur Heart J. 2019; 40(2): 204-212, doi: 10.1093/eurheartj/ehy532, indexed in Pubmed: 30165435.

7. Park DW, Yun SC, Lee JY, et al. C-reactive protein and the risk of stent thrombosis and cardiovascular events after drug-eluting stent implantation. Circulation. 2009; 120(20): 1987-1995, doi: 10.1161/CIRCULATIONAHA.109.876763, indexed in Pubmed: 19884467.

8. Hwang SJ, Park KW, Kwon DA, et al. Korea Stent Thrombosis Investigators. High plasma interleukin-6 is associated with drug-eluting stent thrombosis: possible role of inflammatory cytokines in the development of stent thrombosis from the Korea Stent Thrombosis Registry. Circ J. 2011; 75(6): 1350-1357, doi: 10.1253/circj.cj-10-0488, indexed in Pubmed: 21498913.

9. Katayama T, Nakashima $\mathrm{H}$, Takagi $\mathrm{C}$, et al. Predictors of sub-acute stent thrombosis in acute myocardial infarction patients following primary coronary stenting with bare metal stent. Circ J. 2006; 70(2): 151-155, doi: 10.1253/circj.70.151, indexed in Pubmed: 16434807.

10. Wiviott SD, Braunwald E, McCabe CH, et al. TRITON-TIMI 38 Investigators. Intensive oral antiplatelet therapy for reduction of ischaemic events including stent thrombosis in patients with acute coronary syndromes treated with percutaneous coronary intervention and stenting in the TRITON-TIMI 38 trial: a subanalysis of a randomised trial. Lancet. 2008; 371(9621): 1353-1363, doi: 10.1016/S0140-6736(08)60422-5, indexed in Pubmed: 18377975

11. Steg PG, Harrington RA, Emanuelsson $\mathrm{H}$, et al. PLATO Study Group. Stent thrombosis with ticagrelor versus clopidogrel in patients with acute coronary syndromes: an analysis from the prospective, randomized PLATO trial. Circulation. 2013; 128(10): 1055-1065, doi: 10.1161/CIRCULATIONAHA.113.002589, indexed in Pubmed: 23900047.

12. Armstrong EJ, Sab S, Singh GD, et al. Predictors and outcomes of recurrent stent thrombosis: results from a multicenter registry. JACC Cardiovasc Interv. 2014; 7(10): 1105-1113, doi: 10.1016/j. jcin.2014.05.017, indexed in Pubmed: 25341707.

13. Shimada YJ, Bansilal S, Wiviott SD et al. PLATO Investigators, PLATO study group, PLATO Study Group, PLATO investigators, PLATO Investigators. Ticagrelor versus clopidogrel in patients with acute coronary syndromes. N Engl J Med. 2009; 361(11): 1045-1057, doi: 10.1056/NEJMoa0904327, indexed in Pubmed: 19717846.

14. Armstrong EJ, Feldman DN, Wang TY, et al. Clinical presentation, management, and outcomes of angiographically documented early, late, and very late stent thrombosis. JACC Cardiovasc Interv. 2012; 5(2): 131-140, doi: 10.1016/j.jcin.2011.10.013, indexed in Pubmed: 22361596. 